\title{
Isoquercitrin promotes peripheral nerve regeneration through inhibiting oxidative stress following sciatic crush injury in mice
}

\author{
Jiaying Qiu ${ }^{1,2}$, Xiaoming Yang ${ }^{2}$, Lingbin Wang ${ }^{2}$, Qiuyu Zhang ${ }^{2}$, Wenjing Ma ${ }^{2}$, Ziwei Huang ${ }^{2}$, \\ Yuhua Bao ${ }^{3}$, Lou Zhong ${ }^{3}$, Hualin Sun ${ }^{2}$, Fei Ding ${ }^{1,2}$ \\ ${ }^{1}$ School of Biology and Basic Medical Sciences, Medical College of Soochow University, Suzhou 215123, China; Key Laboratory of Neuroregeneration \\ of Jiangsu and Ministry of Education, Jiangsu Clinical Medicine Center of Tissue Engineering and Nerve Injury Repair, Co-Innovation Center of \\ Neuroregeneration, Nantong University, Nantong 226001, China; ${ }^{3}$ Affiliated Hospital of Nantong University, Nantong University, Nantong 226001, China \\ Contributions: (I) Conception and design: H Sun, F Ding; (II) Administrative support: None; (III) Provision of study materials or patients: None; \\ (IV) Collection and assembly of data: Z Lou, Q Zhang, W Ma; (V) Data analysis and interpretation: J Qiu, Q Zhang, W Ma, Z Huang, X Yang; (VI) \\ Manuscript writing: All authors; (VII) Final approval of manuscript: All authors. \\ Correspondence to: Dr. Hualin Sun; Professor Fei Ding. Key Laboratory of Neuroregeneration of Jiangsu and Ministry of Education, Nantong \\ University, 19 Qixiu Road, Nantong 226001, China. Email: sunhl@ntu.edu.cn; dingfei@ntu.edu.cn.
}

Background: Oxidative stress has been recognized to play a crucial role in the pathogenesis of peripheral nerve injury. Isoquercitrin (quercetin-3-glucoside) is a flavonoid that exhibited many biological activities, including antioxidative effect. However, it is unclear whether isoquercitrin has protective effects on peripheral nerve injury.

Methods: Mice treated by isoquercitrin were used as a case group, and mice injected with saline was the control group. Sciatic behavioral function was assessed using SFI and CMAPs were measured by electrophysiology. Schwann cells proliferation and migration were tested using EdU staining and Transwell migration chambers respectively. The expression of oxidative stress related factors were detected by qRT-PCR and Western blotting.

Results: In present study, our results demonstrated that isoquercitrin $(20 \mathrm{mg} / \mathrm{kg} / \mathrm{day})$ treatment achieved significantly higher SFI and higher amplitude of CMAP, promoted the nerve regeneration and remyelination, increased the production of GAP43, NF200, MAG and PMP22, alleviated target muscle atrophy and autophagy, and suppressed the expression of ATG7, PINK1 and Beclin1 in soleus muscles after sciatic nerve crush. In vitro studies found that isoquercitrin promoted the axonal regeneration of DRGs neurons, the proliferation and migration of Schwann cells, and the expression of proliferating cell nuclear antigen (PCNA) in Schwann cells. The administration of isoquercitrin at 40 and $320 \mu \mathrm{M}$ showed a dose dependent, and high doses of isoquercitrin (160 and $320 \mu \mathrm{M})$ showed better performance in promoting axonal regeneration of DRGs neurons, and the proliferation and migration of Schwann cells than low dose of isoquercitrin (40 $\mu M)$. Furthermore, isoquercitrin significantly inhibited oxidative stress through reducing the production of Nox4 and Duox1, and promoting the expression of Nrf2 and SOD2 in soleus muscles after sciatic nerve crush.

Conclusions: Isoquercitrin may promote motor functional recovery and nerve regeneration following peripheral nerve injury though inhibition of oxidative stress, which highlighted the therapeutic values of isoquercitrin as a neuroprotective drug for peripheral nerve repair applications.

Keywords: Peripheral nerve injury; oxidative stress; isoquercitrin; nerve regeneration

Submitted Jun 27, 2019. Accepted for publication Oct 24, 2019.

doi: $10.21037 /$ atm.2019.11.18

View this article at: http://dx.doi.org/10.21037/atm.2019.11.18 


\section{Introduction}

Peripheral nerve injury is a common clinical problem undergoing various situations, such as sharp instruments, firearm, stretch, compression and ischemia, and iatrogenic injury, which is characterized by the disruption of myelin sheaths and axons $(1,2)$. Different from central nervous system (CNS), the peripheral nervous system (PNS) has been provided with a considerable capacity for spontaneous regeneration in response to traumatic injury (3-5). Following sciatic nerve injury, Schwann cells dedifferentiate and proliferate, migrate into injury site eliminating myelin debris together with macrophages, forming bands of Büngner that provide pathways for regenerating axons (6). However, it will take a long time before the regenerated axons reaching target organs, and the sciatic nerve function scarcely returns to pre-injury levels, consequently resulting in severe loss of function and disability due to the recovery without intervention being gradual and incomplete. Therefore, new treatments and effective drugs are needed to enhance nerve regeneration and improve functional outcomes after injury.

Peripheral nerve injury is accompanied with oxidative stress and inflammation, which have been believed to play an important role in the pathogenesis of peripheral nerve injury (7-11). Oxidative stress is considered to be one of the main origins of neural damage after injury, and play a negative role in nerve functional recovery after peripheral nerve injury (12-14). Diverse methods of peripheral nerves injury can lead to different degrees of oxidative stress (15). Recent studies have shown that inhibiting oxidative stress after peripheral nerve injury could accelerate the repair process and improve the functional recovery after nerve injury $(12,16,17)$. Following peripheral nerve injury, rapid pro-inflammatory response is required for removal of tissue scraps and valid nerve regeneration (13). If the inflammation persists and is not effectively suppressed in a timely manner, functional recovery will be affected. Dampening inflammatory responses contributes to functional recovery from peripheral nerve injury (18). In conclusion, suppressing oxidative stress and inflammation may be an important approach in preventing oxidative and inflammatory neural damage and contributing to nerve regeneration and functional reconstruction after peripheral nerve injury.

Isoquercitrin (quercetin-3-glucoside), a flavonoid compound that is widely distributed in medicinal and dietary plants, displays many biological activities, including antioxidant and inflammation inhibition activities (19,20). It has been reported that isoquercitrin exhibited ROS scavenging activity to protect MSCs against ROS-induced oxidative damage (21), as well as suppressed the production of ROS induced by free fatty acids/lipopolysaccharides within hepatocytes (22). Isoquercitrin could protect liver from acetaminophen induced injury though inhibition of oxidative stress (23). Recent studies have shown that isoquercitrin plays an important role in the protection of CNS injury, such as protecting primary culture of rat cortical neuronal cells from oxygen-glucose deprivationreperfusion induced cell damage (24), and ameliorating cerebral impairment in focal ischemia through antioxidative effect (25). However, the protective effect of isoquercitrin on PNS injury remains unclear.

Therefore, the current study is designed to probe the effect of isoquercitrin on nerve regeneration and functional recovery in a mice model of sciatic nerve crush injury in vivo, explore the effect of isoquercitrin on the proliferation and migration of Schwann cells in vitro, as well as elucidate the effect of isoquercitrin on oxidative stress. Finally, it is possible for us to explore the neuroprotective effects of isoquercitrin on peripheral nerve injury and the potential molecular mechanism.

\section{Methods}

\section{Animals and treatment}

The whole study protocol is approved by the Ethics Committee of Nantong University (No. 20160308-009) and all the procedures were done according to the guidelines of the Animal Care Committee from the Nantong University, Jiangsu Province, China. Male ICR mice (about 20 grams) are maintained in an enriched environment with a room-controlled temperature, $12 \mathrm{~h}$ light-dark cycle, food and water available. ICR mice are anaesthetized before the sciatic nerve exposed, and a 3-mm long nerve in the mid-thigh of hind limb is crushed three times (10 seconds/each time, 3 seconds interval) by use of a hemostatic forceps (26). The ICR mice are randomly grouped into three different groups: control group (Ctrl) represents the sham-operated group; saline group (Saline) represents sciatic nerve crush + intraperitoneal injection of equivalent saline; isoquercitrin group (ISO) represents sciatic nerve crush + intraperitoneal injection of isoquercitrin $(20 \mathrm{mg} / \mathrm{kg} / \mathrm{day})$ after nerve crush. The dose of isoquercitrin is chosen based on previous studies which could get a protective role in disease models $(27,28)$. 


\section{Primary culture of Schwann cells and treatment}

Neonatal 1-day-old Sprague-Dawley (SD) rats are provided from the Experiment Animal Center of Nantong University. Primary SCs are isolated from sciatic nerve of rats. In order to remove the fibroblasts, purifying the mussy Schwann cells with anti-Thy1.1 antibody (Sigma, St Louis, MO) and rabbit complement (Sigma, St Louis, MO). The collected primary Schwann cells are cultured in Dulbecco's modified eagle medium containing 10\% fetal bovine serum (Gibco, Grand Island, NY) at $37^{\circ} \mathrm{C}$ in a humidified incubator with $5 \% \mathrm{CO}_{2}$. Making certain the purity of Schwann cells over $95 \%$, and the cells are passaged for no more than three times prior to use. The purified cells are replanted in 6-well plates for $24 \mathrm{~h}$, and the negative control group is subjected to fasting (nutritional deprivation, ND) for $12 \mathrm{~h}$ in amino acid free and serum free Hank's balanced salt solution (HBSS), while experimental groups are treated with different concentration of isoquercitrin ranging from 40 to $320 \mu \mathrm{M}$, and positive control group is treated with NGF $(50 \mathrm{ng} / \mathrm{mL})$ at the same time. After treatment, the Schwann cells are prepared for related detection.

\section{Western blotting}

Cultured Schwann cells and sciatic nerves are lysed in RIPA lysis buffer from Beyotime Biotechnology (Haimen, China) and the quantification of whole sample lysates is performed using BCA Protein Quantification Kit (Beyotime Biotechnology, Haimen, China). Equivalent amounts of isolated protein were separated on 10\% SDS-PAGE and transferred to PVDF membranes (Millipore, Bedford, MA, USA). Then, these membranes were blocked with $5 \%$ nonfat dry milk in Tris- $\mathrm{HCl}$ buffered saline (TBS) at room temperature and probed with GAP43 (1:1,000) (Invitrogen Antibodies, Waltham, MA), NF200 (1:1,000) (Invitrogen Antibodies, Waltham, MA), MAG $(1: 2,000)$ (LifeSpan BioSciences, Seattle, WA), PMP22 (1:2,000) (LifeSpan BioSciences, Seattle, WA), PCNA $(1: 1,000)$ (LifeSpan BioSciences, Seattle, WA), $\beta$-actin $(1: 2,000)$ (LifeSpan BioSciences, Seattle, WA), Nox4 $(1: 2,000)$ (LifeSpan BioSciences, Seattle, WA), Nrf2 (1:2,000) (LifeSpan BioSciences, Seattle, WA), ATG7 $(1: 2,000)$ (Invitrogen Antibodies, Waltham, MA), PINK1 (1:1,000) (LifeSpan BioSciences, Seattle, WA), Beclin1(1:2,000) (Biorbyt, San Francisco, CA) and SOD2 (1:2,000) (Biorbyt, San Francisco, CA). Next day, following been washed with TBST, these membranes are incubated with the corresponding HRPconjugated secondary antibodies. Blotted bands are visualized with enhanced chemiluminescent solution (Thermo Fisher Scientific, Inc., Carlsbad, CA) and exposed on films.

\section{qRT-PCR}

Total RNA is extracted using the RNeasy kit (Qiagen, Valencia, CA). The cDNA is synthesized using the first-strand cDNA synthesis kit with oligo dT primers (Invitrogen, Carlsbad, CA). The following primers used in this study are prepared by the Shanghai Generay Biotech Co., Ltd., (Shanghai, China): mouse Duox1 (GI: 150010670) left primer 5'-CCTGGGCCACTGAAGTTTTC-3', right primer 5'-GGAGAGTGCAGGGTTGATGT-3'; mouse Nrf2 (GI: 20073087 ) left primer 5 ' - GTTGCCCACATTCCCAAACA-3', right primer 5'-CTGATGAGGGGCAGTGAAGA-3'; mouse SOD2 (GI: 76253932 ) left primer 5 ' - CCAGACCTGCCTTACGACTA-3 ', right primer 5'-TGAAGAGCGACCTGAGTTGT-3'; mouse GAPDH (GI: 193423) left primer 5'-AACTTTGGCATTGTGGAAGG-3', right primer 5'-ACACATTGGGGGTAGGAACA-3'. All RT-qPCR were performed using QuantiNova SYBR Green kit (QIAGEN, 208054) with primers on an Applied Biosystems StepOne realtime PCR System to evaluate the mRNA expression of the corresponding genes. The relative expression is measured using the $2^{-\Delta \Delta \mathrm{Ct}}$ method (29). Relative expression is calculated from cycle threshold values corrected for GAPDH.

\section{Behavioral analysis}

Sciatic behavioral function is assessed using the sciatic functional index (SFI). The SFI is a generally used test to assess behavioral recovery and return of function after sciatic nerve injury (30). Briefly, the hind paws of each animal are moistened with chemically synthesized red paint and the animals are allowed to walk unassisted along a $6 \times 60 \mathrm{~cm}^{2}$ corridor lined with white paper. The prints chosen for measurements are complete. The tracks are evaluated for two different parameters: toe spread (the distance between first and fifth toes) and print length (the distance between the third toe and the hind pad). Measurement of each parameter is made for the right 
(normal) and the left (experimental) paw prints. Using the parameters, preoperative and postoperative SFI for each animal is determined according to our previous study (26).

\section{Electrophysiological measurements}

ICR mice are anaesthetized before the left mid-thigh sciatic nerve exposed. The previous surgical site is reopened and the sciatic nerve is exposed again. Electrical stimuli $(10 \mathrm{mV})$ are applied to the sciatic nerve trunk at the proximal and distal ends of the injured site sequentially. Compound muscle action potentials (CMAPs) are recorded on the gastrocnemius belly. The same assessments are conducted at the uninjured contralateral side.

\section{Cell proliferation assay}

The proliferation rate of Schwann cells is measured with EdU staining (Ribobio, Guangzhou, China). Cultured primary Schwann cells are suspended in the complete medium prewarmed to $37^{\circ} \mathrm{C}$, and seeded at a density of $2 \times 10^{5}$ cells $/ \mathrm{mL}$ on $96-$ well plates coated with poly-L-lysine. Schwann cells are incubated with $100 \mu \mathrm{M} \mathrm{EdU} \mathrm{and} \mathrm{grew} \mathrm{for}$ 24 h. Cell cultures were fixed with $4 \%$ paraformaldehyde and DAPI is added and slides are visualized under a fluorescent microscope. The total number of Schwann cells for each image is determined by counting the number of DAPI-counterstained blue nuclei, while the total number of proliferating SCs is measured by counting the number of Edu-positive red nuclei. The percent of proliferation is calculated by the ratio of total EdU-positive nuclei to total number of cells.

\section{Cell migration assay}

Cell migration assay is tested using Transwell migration chambers which are 8-um pore size (Corning, NY, USA). The bottom chambers are filled with $600 \mu \mathrm{L}$ medium with different concentrations of isoquercitrin ranging from 40 to $320 \mu \mathrm{M}$. Schwann cells $\left(1 \times 10^{5} / \mathrm{mL}\right)$ are seeded into the top chambers in $100 \mu \mathrm{L}$ medium. After incubated in a humidified $5 \% \mathrm{CO}_{2}$ for $24 \mathrm{~h}$ at $37{ }^{\circ} \mathrm{C}$, Schwann cells transferred to the lower chamber are stained with $0.5 \%$ crystal violet at room temperature about $20 \mathrm{~min}$, while the Schwann cells on the upper chamber are wiped. The migration rate is calculated by means of randomly selected image fields.

\section{Statistical analysis}

All statistical analyses are conducted with a SPSS Software Version 17.0 (SPSS Inc., Chicago, IL). All data are expressed as the means \pm SEM. Results are analyzed by One-way ANOVA with a subsequent Bonferroni's test for pairwise comparisons. $\mathrm{P}<0.05$ is considered statistically significant.

\section{Results}

Isoquercitrin improves the recovery of motor function after sciatic nerve crush

To evaluate the effects of treatment with isoquercitrin on the recovery of motor function after sciatic nerve crush, the sciatic functional index (SFI), an index of functional nerve reconstruction, is used in this study. The SFI value varies from 0 to -100 , with 0 and -100 indicating normal function and absolutely loss of motor function, respectively. In current study, the mice from the saline treated group showed a gradually increase in SFI value, which suggested a spontaneous recovery of motor function (Figure 1A). Similarly, the mice from the ISO treated group also showed a gradually increase in SFI value, at 11 and 15 days after injury, the SFI values in the ISO treated group were obviously higher than those in the saline treated group $(\mathrm{P}<0.01$, Figure $1 A)$. The SFI values were superior than those in the saline treated group at 19 and 23 days after injury, but there was no statistical significance between them. Therefore, in the following studies, the time at 15 days after injury was chosen to further study.

The recovery of sciatic nerve function is based on the nerve reinnervation of the target muscles. The amplitude of CMAP reflects the number of nerve fibers reinnervating target muscles, which is usually used to evaluate the extent of motor functional recovery. At 15 days after injury, ISO treated group accomplished markedly higher amplitude of CMAP than the saline treated group $(\mathrm{P}<0.05$; Figure $1 B, C)$, which demonstrated that a better recovery in the motor function was obtained in the ISO treated group following sciatic nerve crush.

\section{Isoquercitrin is beneficial for remyelination and nerve regeneration after peripheral nerve injury}

To evaluate the effects of treatment with isoquercitrin on the remyelination after sciatic nerve crush, transmission 
A
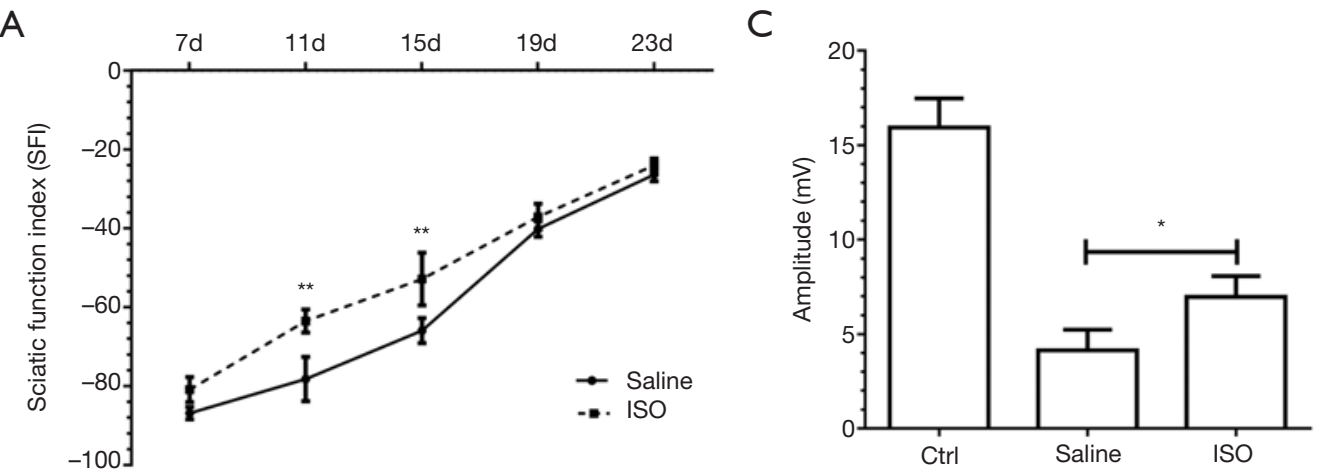

B

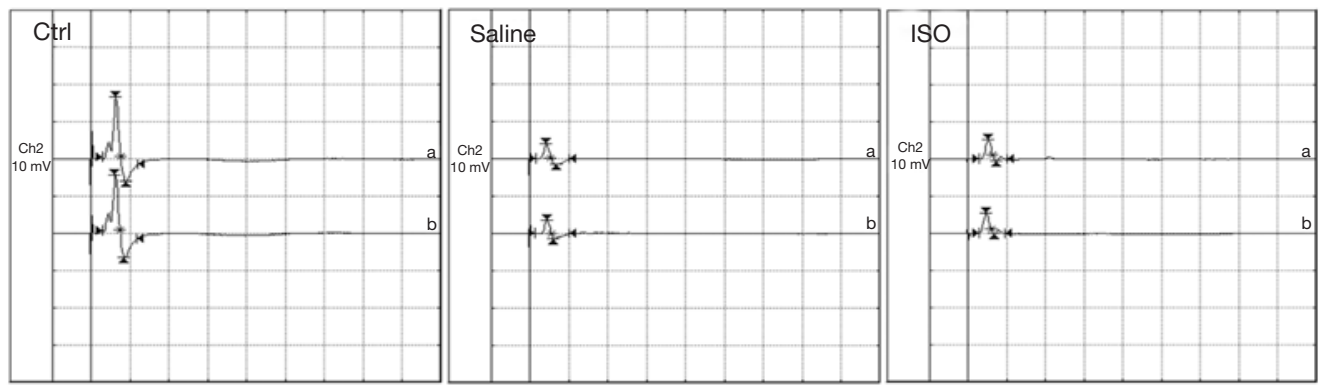

Figure 1 Effects of ISO on the recovery of motor function after sciatic nerve crush. (A) Effects of ISO on the SFI after sciatic nerve crush. (B) CMAP measurements at 15 days after sciatic nerve crush and representative traces of CMAP. (C) Effects of ISO on the CMAPs after sciatic nerve crush. Ctrl, normal control group; Saline, saline treated group; ISO, ISO treated group. * $\mathrm{P}<0.05 ;{ }^{* *}, \mathrm{P}<0.01$.

electron is used to observe the mean thickness of myelin sheath and the mean number of myelin sheath layers in current study. The mean thickness of myelin sheath and the mean number of myelin sheath layers all displayed significant reduction in saline treated group compared to normal control group, while the treatment with isoquercitrin could rescue the reduction of the mean thickness of myelin sheath and the mean number of myelin sheath layers induced by the treatment with saline $(\mathrm{P}<0.05$, Figure $2 A, B, C)$, which suggested that isoquercitrin promoted the remyelination after sciatic nerve crush.

Subsequently, we evaluate the effects of isoquercitrin on the Myelin formation associated protein. Therefore, we evaluate the expression of the myelin-associated glycoprotein (MAG) and peripheral myelin protein 22 (PMP22). MAG is implicated in the formation and maintenance of myelin (31) and PMP22 is primarily expressed in the compact myelin and promotes the formation and maintenance of myelin sheaths in the PNS $(32,33)$. The expression of MAG and PMP22 were reduced in saline treated group compared to normal control group, which indicated that the structure and function of myelin sheath had been damaged to some extent. The treatment with isoquercitrin had a conservatory effect in increasing the expression of MAG and PMP22 compared to the treatment with saline $(\mathrm{P}<0.01$, Figure $2 D, E)$. These results demonstrated that isoquercitrin was beneficial to remyelination after peripheral nerve injury.

To elucidate the effects of treatment with isoquercitrin on the nerve regeneration after sciatic nerve crush, we evaluate the effects of treatment with isoquercitrin on the nerve growth associated proteins growth-associated protein 43 (GAP43) and neurofilament 200 (NF200). GAP43 is highly abundant in the neuronal growth cone involved in growth cone guidance and actin cytoskeleton organization during development and regeneration (34) and NF200 is a marker of myelinated neurons (35). Western blot analysis displayed that following the sciatic nerve crush the expression of GAP43 in the saline treated group showed an increase compared to that in the normal control, whereas the treatment with ISO had a better protective effect in enhancing the expression of GAP43 compared to the saline treated group $(\mathrm{P}<0.01)$. The expression of NF200 in the saline treated group displayed significant reduction compared to that in the normal control, whereas the treatment with ISO had a protective effect in increasing 

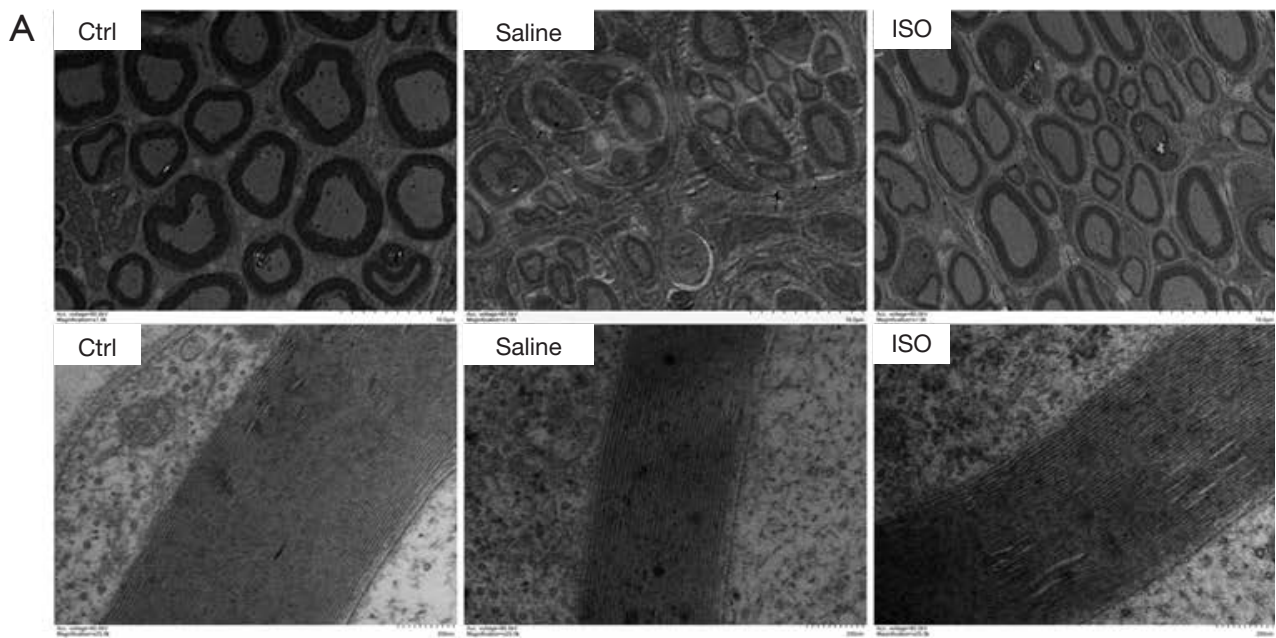

B

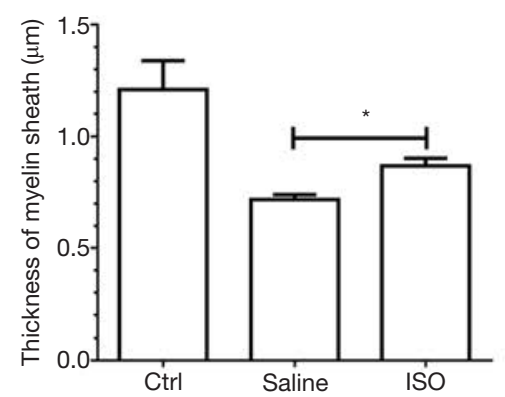

$\mathrm{D}$

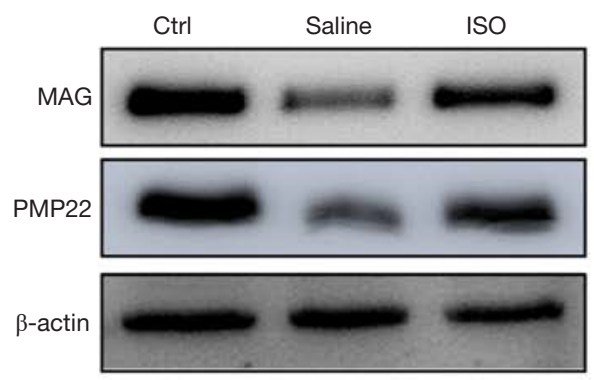

$\mathrm{F}$

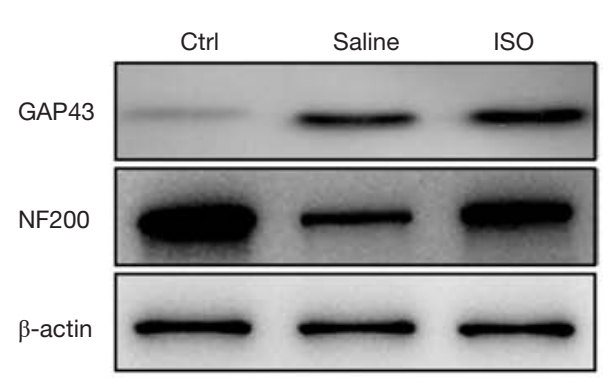

C

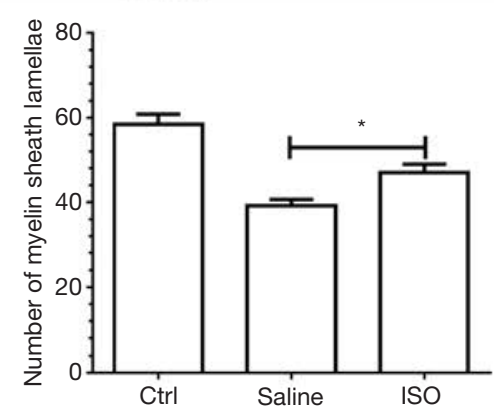

$\mathrm{E}$

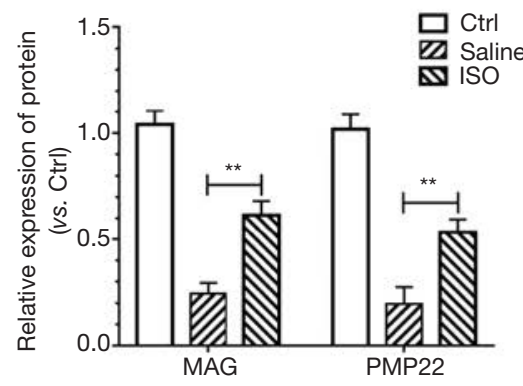

G

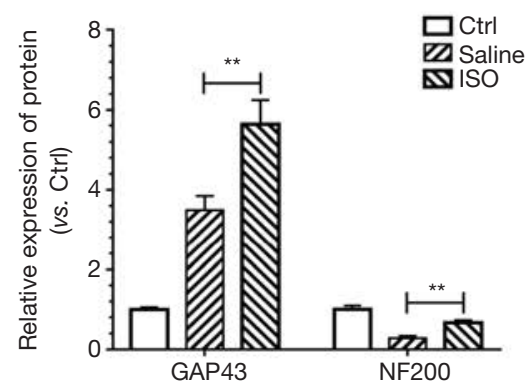

Figure 2 Effects of ISO on the remyelination at 15 days after sciatic nerve crush. (A) The representative transmission electron microscopic images were selected from 3 different groups. (B) Statistical results of the mean thickness of myelin sheath in mice from different groups. (C) Statistical results of the mean number of myelin sheath layers in mice from different groups. (D) The representative images of MAG and PMP22 for western blotting analysis. (E) Statistical results of the relative expression of MAG and PMP22 in mice from different groups. (F) Representative images of GAP43 and NF200 for western blotting analysis. (G) The relative expression of GAP43 and NF200 after sciatic nerve crush. Ctrl, normal control group; Saline, saline treated group; ISO, ISO treated group. * $\mathrm{P}<0.05$; ** $\mathrm{P}<0.01$. 

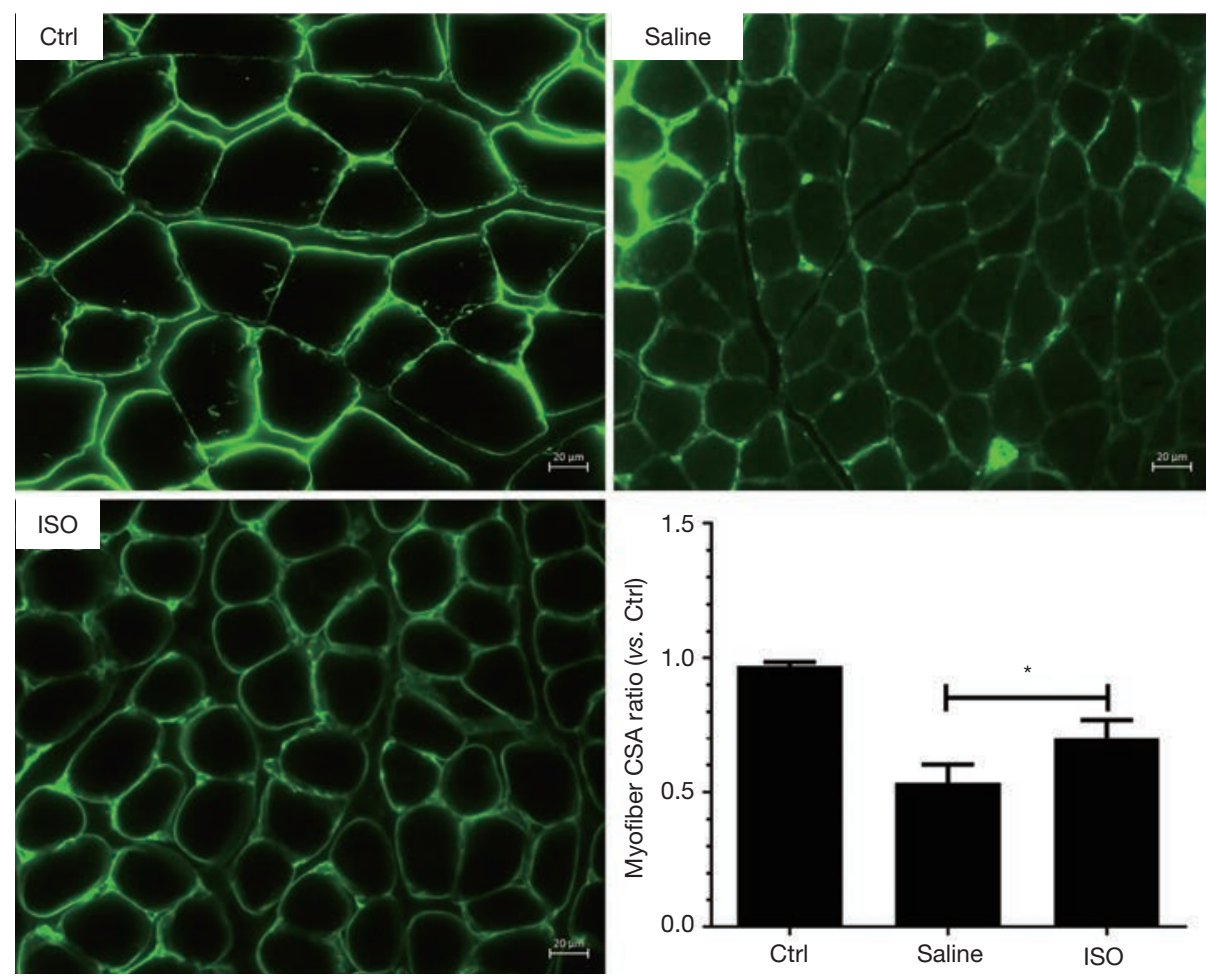

Figure 3 Effects of ISO on muscle atrophy at 15 days after sciatic nerve crush. Soleus muscles of mice were stained for laminin after at 15 days after sciatic nerve crush and the representative laminin staining images were selected from 3 different groups. Statistical results of the myofiber CSA ratio (vs. Ctrl) in mice from different groups. Ctrl, normal control group; Saline, saline treated group; ISO, ISO treated group. *, $\mathrm{P}<0.05$.

the expression of NF200 compared to the saline treated group $(\mathrm{P}<0.01$, Figure $2 F, G)$. These data demonstrated that isoquercitrin promoted the nerve regeneration after sciatic nerve crush.

\section{Isoquercetin alleviates muscle atrophy and inhibits autophagy after sciatic nerve crush}

Isoquercitrin could promote nerve regeneration, myelin formation and nerve function recovery of injured sciatic nerve. Therefore, laminin staining is used to explore the effect of isoquercitrin on muscle cross-sectional area (CSA). Current study showed that the mean CSA of soleus muscles from the mice treated with saline was smaller than that in the mice from normal control group, and the mean CSA of soleus muscles from the mice treated with isoquercitrin was bigger than that in the mice treated with saline $(\mathrm{P}<0.05$, Figure 3$)$. There were many autophagic vesicles or autophages in saline treated group compared to normal control group, while the treatment with isoquercitrin could reduce the production of autophagic vesicle or autophages (Figure $4 A$ ), at the same time accompanied by decreased expression of autophagy-related proteins ATG7, PINK1 and Beclin1 (Figure 4B,C). These data suggested that isoquercitrin could alleviate muscle atrophy and inhibit autophagy of target muscles after sciatic nerve crush.

\section{Isoquercetin promotes axon growth of dorsal root ganglion neurons}

To evaluate the effects of isoquercitrin on axon growth of dorsal root ganglion neurons, the dorsal root ganglions of fetal rats at 14 days of gestation are planted on a 24-well plate for 72 hours, and then the medium is replaced with HBSS in the presence or absence of NGF or isoquercitrin with different concentrations for 24 hours. The dorsal root ganglions are stained with NF200. Results showed that the length of axon extended from DRG explants in isoquercitrin treated group was larger than that in only HBSS treated group. The administration of isoquercitrin at 

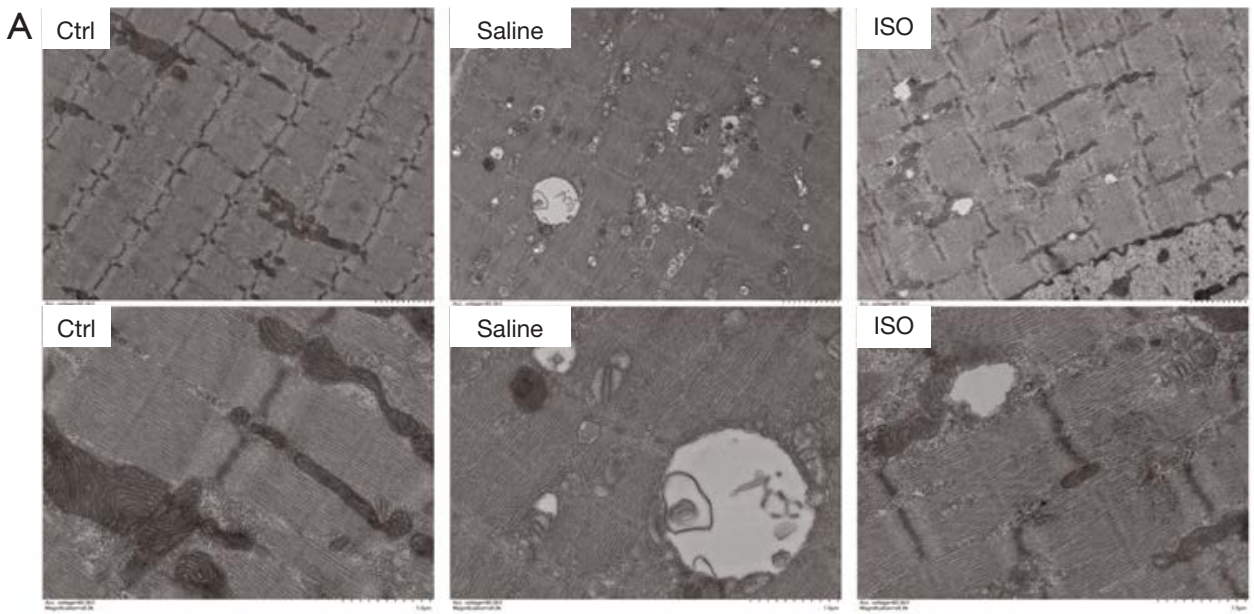

B

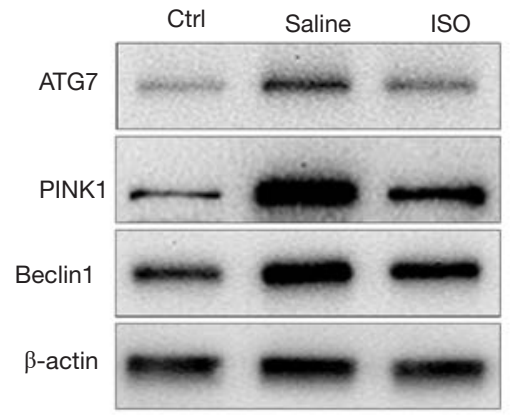

C

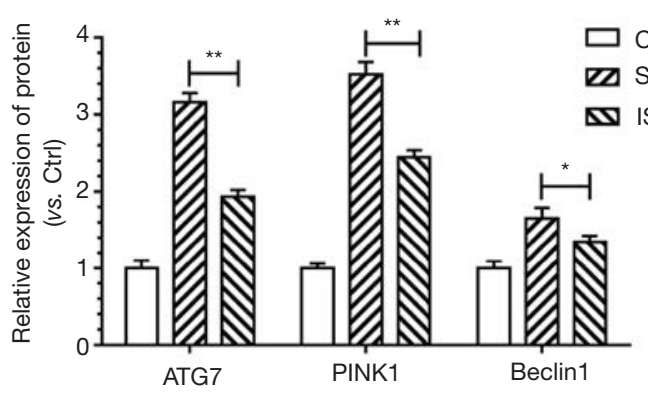

Figure 4 Effects of ISO on muscle autophagy at 15 days after sciatic nerve crush. (A) Soleus muscles of mice were observed by electron microscope at 15 days after sciatic nerve crush and the representative images were selected from 3 different groups. Scale bar, $10 \mu \mathrm{m}$. (B) The representative images of ATG7, PINK1 and Beclin1 for western blotting analysis. (C) The relative expression of ATG7, PINK1 and Beclin1 after sciatic nerve crush. Ctrl, normal control group; Saline, saline treated group; ISO, ISO treated group. *, P<0.05; **, P<0.01.

40 and $320 \mu \mathrm{M}$ showed a dose dependent, and high doses of isoquercitrin $(160$ and $320 \mu \mathrm{M})$ showed better performance in promoting axonal regeneration of DRGs neurons than low dose of isoquercitrin $(40 \mu \mathrm{M})$. Additionally, the number of nerve fiber bundles from DRG explants treated with high doses of isoquercitrin $(160$ and $320 \mu \mathrm{M})$ was also larger than that treated with low dose of isoquercitrin $(40 \mu \mathrm{M})(\mathrm{P}<0.05$, Figure 5). The length and the number of nerve fiber bundles extended from DRG explants were no significant difference between NGF treated group and high doses of isoquercitrin treated group $(160$ and $320 \mu \mathrm{M})$. All these data demonstrated that isoquercitrin promoted an increase in the length and the number of nerve fiber bundles extended from DRG explants, and this effect was getting better and better with the increase of isoquercitrin concentration.

Isoquercetin improves the proliferation of Schwann cells
To evaluate the effects of isoquercitrin on the proliferation of Schwann cells, the cell viability and proliferation ability are analyzed through MTT assay and EDU staining, respectively. Results indicated that HBSS treatment significantly reduced the viability of Schwann cells and isoquercitrin could enhance the viability of Schwann cells, showing a dose dependent $(\mathrm{P}<0.01$, Figure $6 A)$. EDU staining also showed that HBSS significantly decreased the proliferation ability of Schwann cells and isoquercitrin improved the proliferation ability of Schwann cells treated with HBSS, which also showed a dose dependent $(\mathrm{P}<0.01$, Figure 6B,C). Proliferating cell nuclear antigen (PCNA) is known as a molecular marker for proliferation (36). Our data demonstrated that the expression of PCNA was inhibited in HBSS treated group, whereas the treatment 


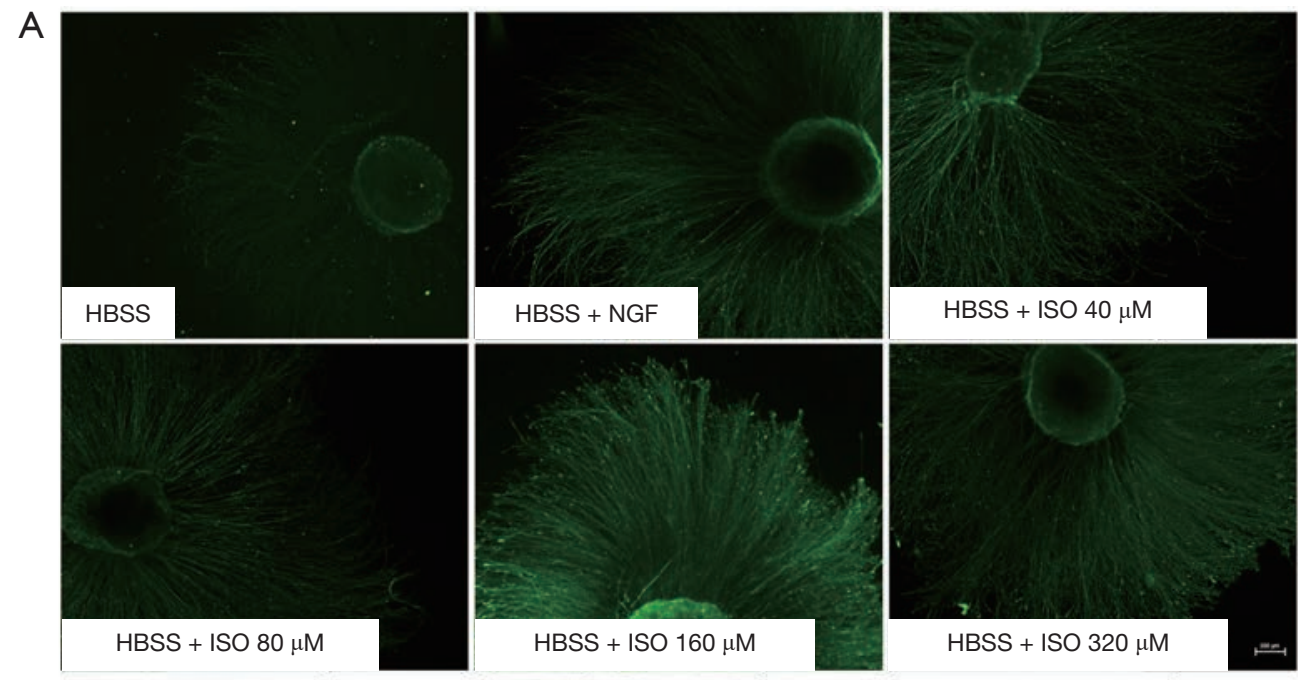

B

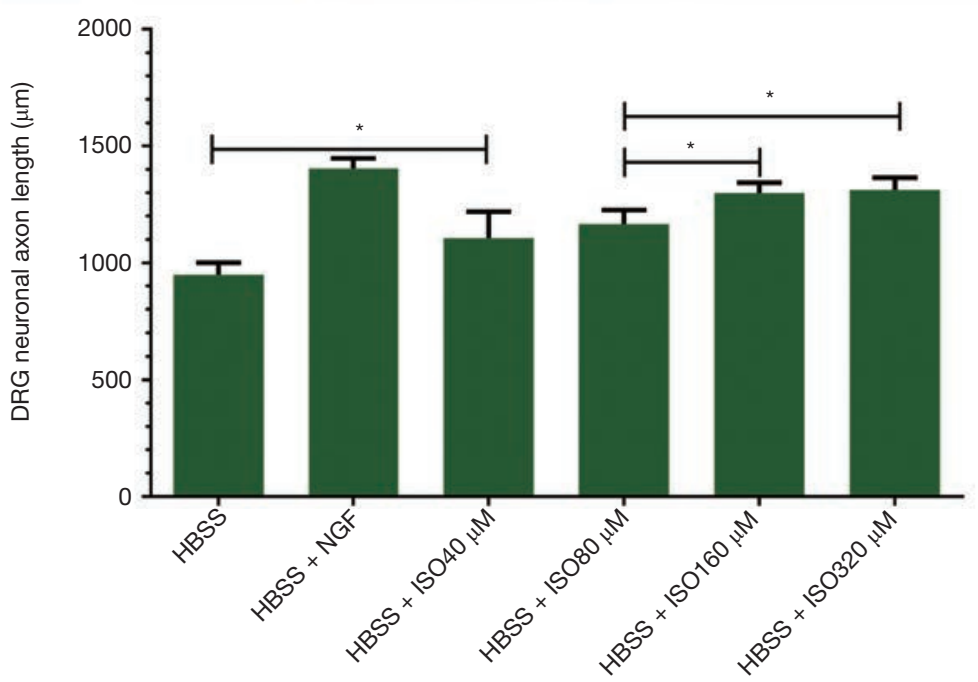

Figure 5 Effects of isoquercetin on axon growth of dorsal root ganglion neurons. (A) The nerve fiber extended from DRG explants were stained with NF200 and the representative images were selected from 6 different groups. Scale bar, $200 \mu \mathrm{m}$. (B) Statistical results of the length of axon extended from DRG explants from different groups. *, $\mathrm{P}<0.05$.

with isoquercitrin had a better protective effect in increasing the expression of PCNA $(\mathrm{P}<0.01$, Figure $6 D, E)$. With the increase of isoquercitrin concentration, the promotion ability became stronger and stronger.

\section{Isoquercetin promotes the migration of Schwann cells}

To evaluate the effects of isoquercitrin on the migration of Schwann cells, the transwell chamber is used to analyze the ability of Schwann cell migration. Results demonstrated that the ability of Schwann cell migration significantly reduced in HBSS treated group and isoquercitrin could enhance the ability of Schwann cell migration, showing a dose dependent enhancement $(\mathrm{P}<0.01$, Figure $7 A, B)$.

\section{Isoquercetin suppresses oxidative stress after sciatic nerve crush}

Peripheral nerve injury is accompanied with oxidative stress (9-11). To evaluate the effects of treatment with isoquercitrin on oxidative stress after sciatic nerve crush, qRT-PCR and western blotting are used to examine the NADPH oxidase 4 (Nox4), Duox1, SOD2 and nuclear factor E2-related factor-2 (Nrf2). Nox4 and Duox1 could promote the production of $\operatorname{ROS}(37,38)$. Nrf2, a 
Page 10 of 16

A

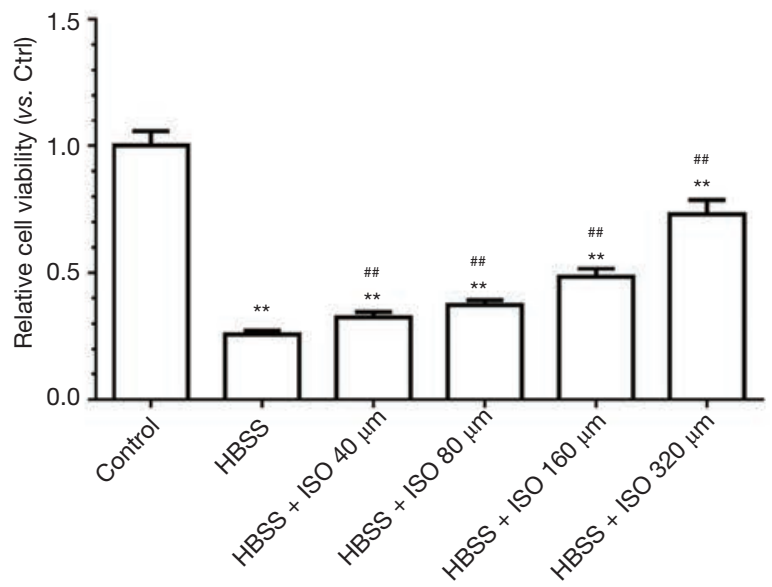

Qiu et al. Isoquercitrin promotes peripheral nerve regeneration

C

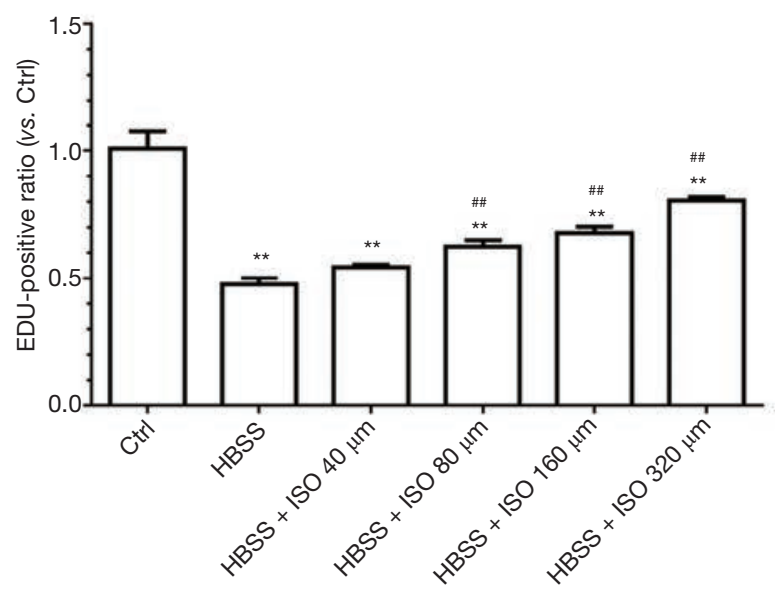

B

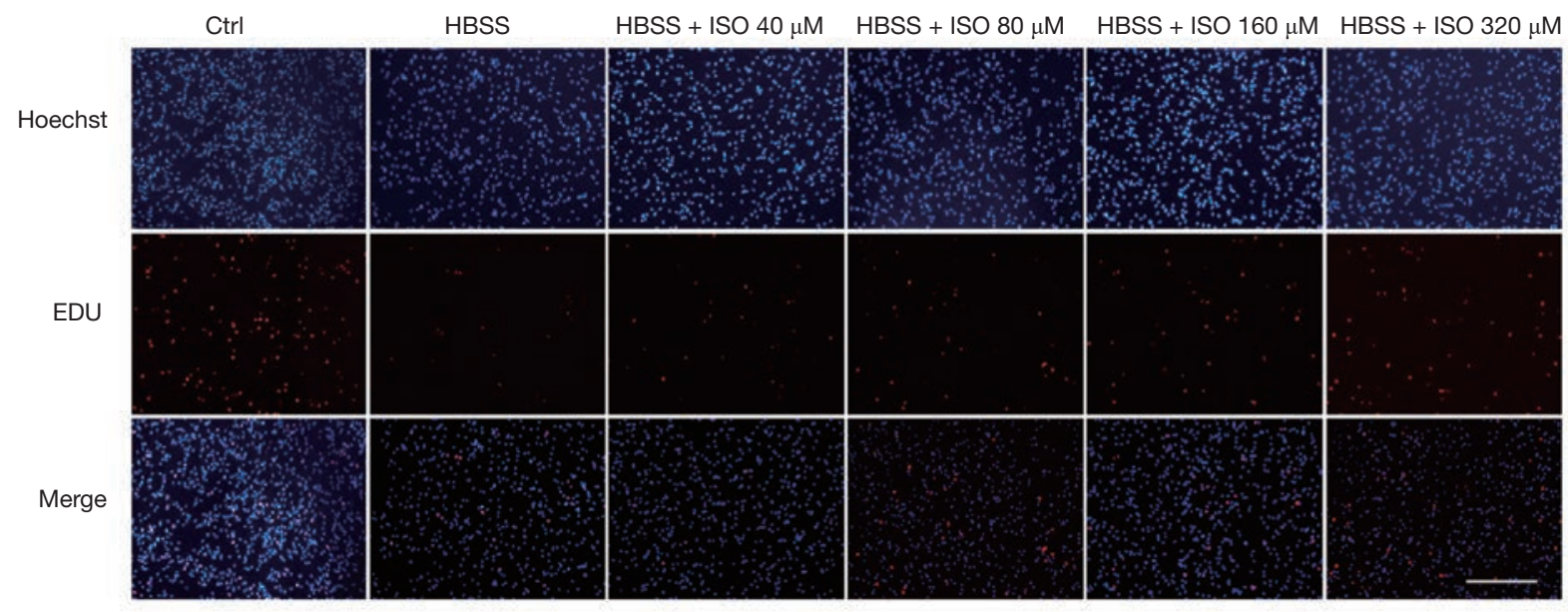

D

E

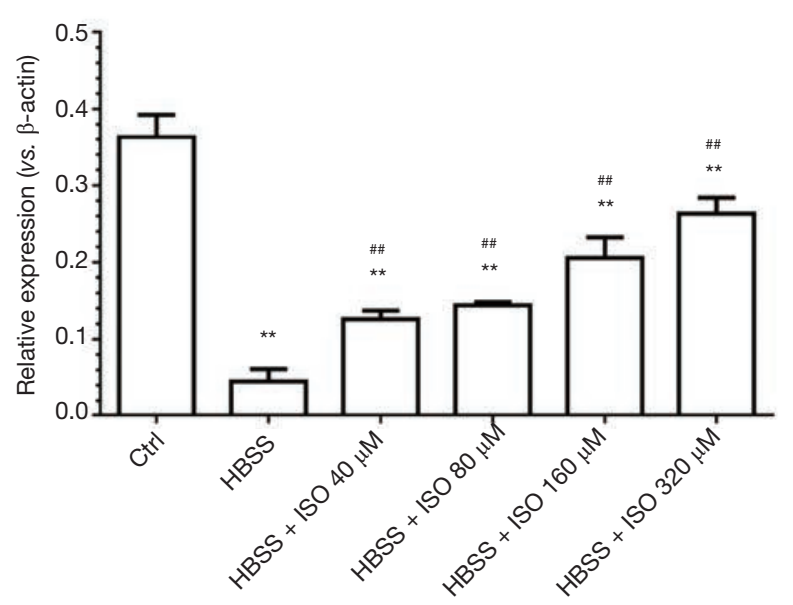

Figure 6 Effects of isoquercetin on the proliferation ability of Schwann cells. (A) The cell viability was determined through MTT assay. (B) The images of EdU staining (red) and Hoechst 33342 staining (blue) were used to measure cell proliferation, scale bar, $400 \mu \mathrm{m}$. (C) Statistical results of the proliferation ability of Schwann cells from 6 different groups. (D) The representative images of PCNA for western blotting analysis. (E) Histograms showing the relative expression of PCNA from different groups. ** $\mathrm{P}<0.01$, vs. Ctrl; ${ }^{\# \#}, \mathrm{P}<0.01, v s$. HBSS. 

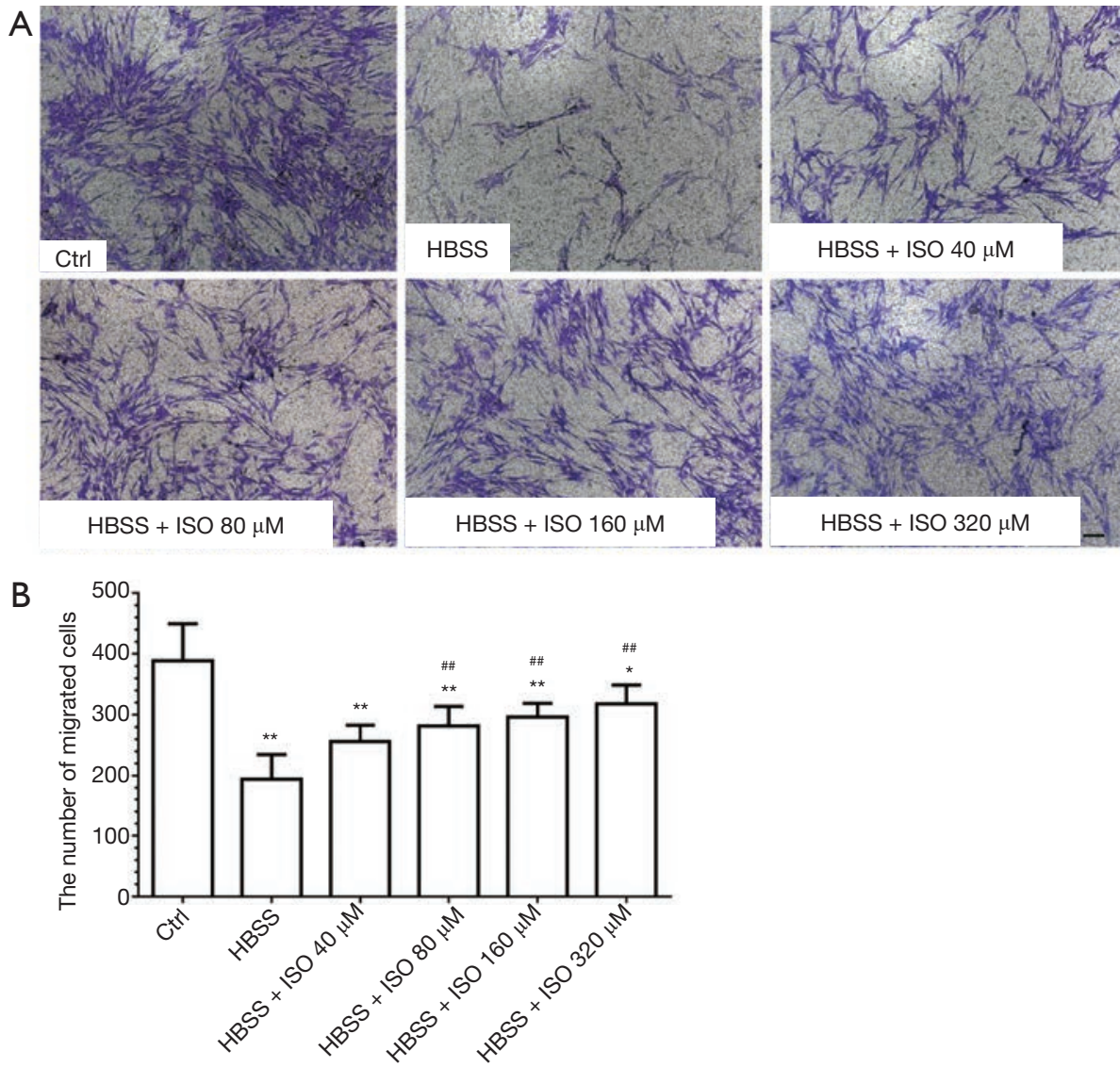

Figure 7 Effects of isoquercetin on the migration ability of Schwann cells. (A) The representative images of crystal violet staining for Schwann cells. (B) Statistical results of the migration ability of Schwann cells from 6 different groups. Scale bar, $50 \mu \mathrm{m}$. *, $\mathrm{P}<0.05$; **, $\mathrm{P}<0.01$, vs. Ctrl; ${ }^{\#,} \mathrm{P}<0.01$, vs. HBSS.

transcription factor, could decrease the production of ROS (39). SOD2 is an enzyme responsible for reducing superoxide radicals in mitochondria (40). Our results demonstrated that isoquercitrin could reduce the production of Nox4 and Duox1, promote the expression of Nrf2 and SOD2 (Figure 8), which indicated that isoquercitrin suppressed oxidative stress after sciatic nerve crush.

\section{Discussion}

Medication is generally used to promote axonal regeneration after peripheral nerve crush injury (41). Vitamin B12, recognized to play a crucial role in neuroprotection, is frequently used in peripheral neuropathy (42). However, side effects of vitamin B12, including diarrhea, skin harassment, rash, allergic reaction and even anaphylactic shock, limit the use of vitamin B12 for the treatment of nerve damage (43). Therefore, it is very important to develop new drugs with little side effects to promote the repair of nerve injury. In current study, we investigated the effects of isoquercitrin on nerve regeneration and functional recovery after nerve crush injury in mice. We found that isoquercitrin significantly improved nerve regeneration and functional recovery in vivo, and promoted the axonal regeneration of DRGs neurons, the proliferation and migration of Schwann cells in vitro. Further investigation showed that isoquercitrin significantly inhibited oxidative stress after sciatic nerve crush. These findings highlighted the therapeutic values of isoquercitrin as a neuroprotective drug for peripheral nerve repair applications.

In the present study, we found that treatment with saline induced a gradual recovery in locomotive activity as evidenced by the SFI value of -30 at 23 days post nerve crush, which suggested a spontaneous recovery of 
A

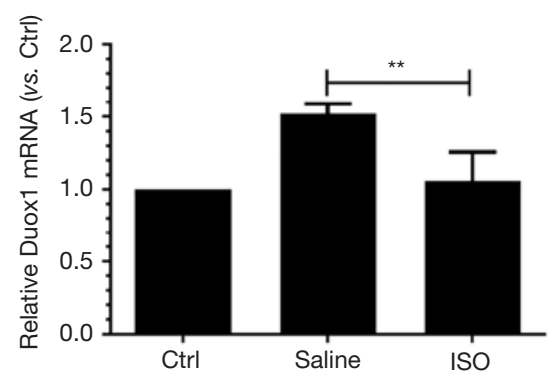

B

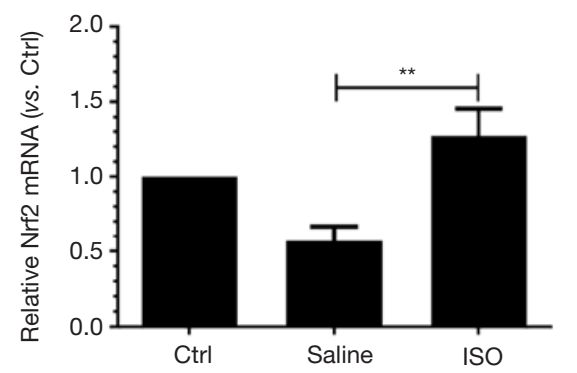

C

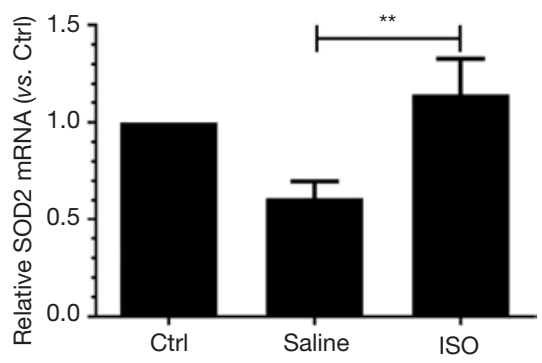

D

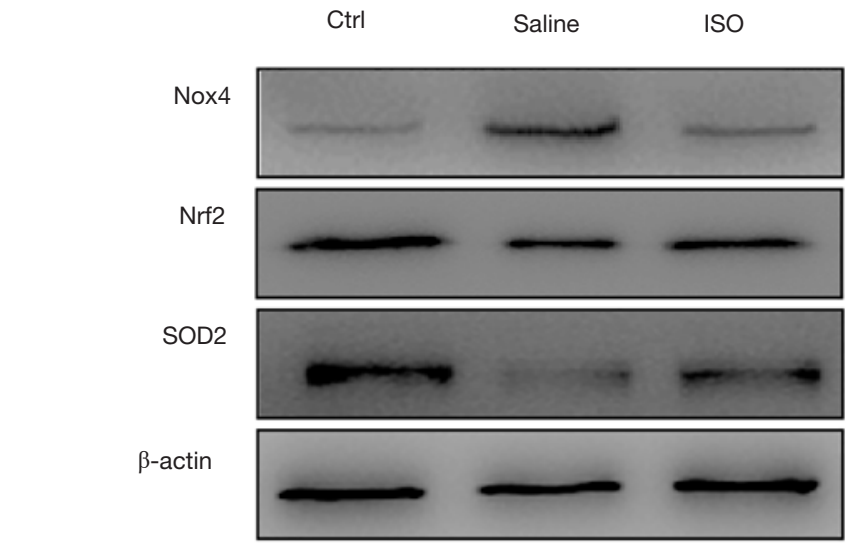

$\mathrm{F}$

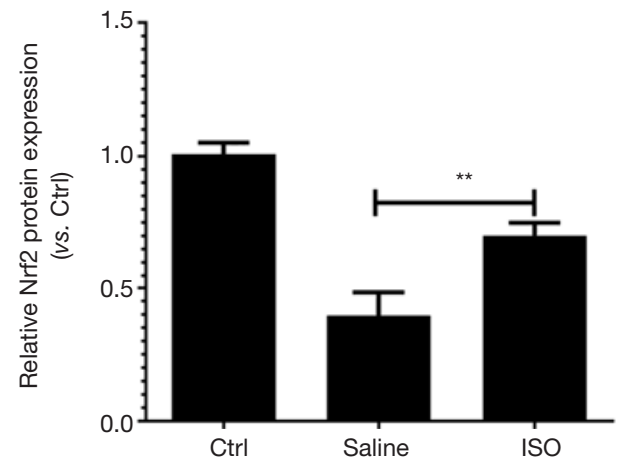

$E$

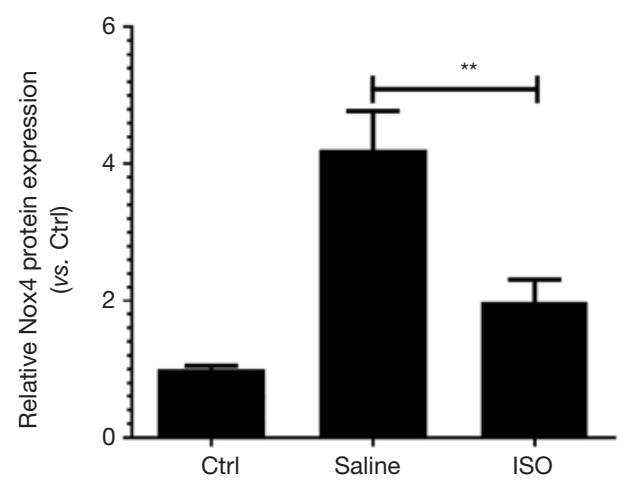

Figure 8 Effects of isoquercetin on oxidative stress after sciatic nerve crush. (A,B,C) The relative mRNA expression of Duox1, Nrf2 and SOD2. (D) The representative images of Nox4, Nrf2 and SOD2 for western blotting analysis. (E,F,G) the relative expression of Nox4, Nrf2 and SOD2 after sciatic nerve crush. Ctrl, normal control group; Saline, saline treated group; ISO, ISO treated group, * $\mathrm{P}<0.05,{ }^{* *}, \mathrm{P}<0.01$. motor function. Treatment with isoquercitrin caused a significant recovery, however, different promotion rates in locomotive activity at 11 and 15 days, which suggested that isoquercitrin could accelerate motor functional recovery during the period from 11 to 15 days post nerve crush, also implied the presence of time window for drug therapy after nerve injury. This is consistent with previous study (44). Thus, in the following studies, the time at 15 days after nerve injury was chosen to further study. The recovery of sciatic nerve function is based on the nerve reinnervation of the target muscles, as well as the amplitude of CMAP, as markers of axon integrity, reflecting the number of nerve 
fibers reinnervating target muscles (45). At 15 days after injury, treatment with isoquercitrin achieved significantly higher amplitude of CMAP than the saline treated group, which demonstrated that isoquercitrin may promote the nerve reinnervation of target muscles, thereby improving the amplitude of CAMP following sciatic nerve crush.

The morphological features of target muscle and regenerated nerve fibers can reflect the degree of functional recovery after peripheral nerve injury (46). Our study showed that the functional performances of isoquercitrintreated mice were concomitant with the morphometric analysis of target muscles. Treatment with isoquercitrin significantly improved the histomorphologic appearance of target muscles, and enlarged the mean diameter and thickness of myelin sheath in regenerated nerve fibers after nerve injury. These current evidences that isoquercitrin was capable of heightening axonal outgrowth and increasing functional recovery.

GAP43, a PKC-activated phosphoprotein, is often implicated in neurite outgrowth during development and axon regeneration (47) and NF200 is a marker of myelinated neurons (35). Our data showed that isoquercitrin had a protective effect in increasing the expression of GAP43 and NF200 compared to the saline treated group, which suggested that isoquercitrin promoted the nerve regeneration via enhancing the expression of GAP43 and NF200 after sciatic nerve crush. In addition, Palazzolo et al. found that isoquercitrin promoted neurite elongation via stimulating extensive neurites enriched in the synaptic vesicle protein synaptotagmin-1 (48). Our data also found that isoquercitrin can promote the axonal outgrowth of DRG neurons in vitro, which may be related to its nerve protection through stimulating extensive neurites enriched to promote the axonal outgrowth. The precise mechanism needs further experimental confirmation.

Myelination of the PNS is essential for axonal function (49). The enhanced axonal growth and regeneration by isoquercitrin was also supported by the enlarged diameter and thickness of myelin sheath induced by treatment with isoquercitrin. MAG involves in myelinmaintenance and PMP22, primarily expressed by Schwann cells, contributes to the formation and maintenance of myelin sheaths $(32,33,50)$. Our data also demonstrated that isoquercitrin promoted the expression of MAG and PMP22 compared to saline, which suggested that isoquercitrin was beneficial to remyelination via promoting the expression of MAG and PMP22 after peripheral nerve injury. Moreover, Schwann cell proliferation and migration contribute to nerve repair following peripheral nerve injury (51). The proliferative Schwann cells can not only secrete neurotrophic factors to promote nerve regeneration, but also migrate to injured sites to promote remyelination post peripheral nerve injury (52). Our results suggested that isoquercitrin can not only promote the proliferation of Schwann cells, but also improve the migration of Schwann cells, which indicated that isoquercitrin was beneficial for the axonal outgrowth and remyelination through promoting the proliferation and migration of Schwann cells.

Functional and morphological recovery of target muscle is direct evidence of nerve regeneration and reinnervation (53). Peripheral nerve injuries frequently induce muscle atrophy through enhancing the induction of proteolysis system, such as autophagy-lysosome and ubiquitin-proteasome systems $(54,55)$. Our data demonstrated that the mean CSA of soleus muscles from the mice treated with isoquercitrin was bigger than that in the mice treated with saline, which indicated that isoquercitrin could alleviate muscle atrophy after sciatic nerve crush. Furthermore, our results also showed that treatment with isoquercitrin suppressed the activation of autophagy in soleus muscles.

Peripheral nerve injury is accompanied with oxidative stress and inflammation (9-11). Oxidative stress is defined to be one of the main factors of neural damage after injury $(13,14)$. Recent studies have shown that inhibiting oxidative stress after peripheral nerve injury could accelerate the repair process and improve the functional recovery after nerve injury $(12,16,17)$. However, whether isoquercitrin is capable of suppressing oxidative stress to promote nerve repair and functional recovery remains to be elucidated. Our results revealed that isoquercitrin can suppress oxidative stress via reducing the production of Nox4 and Duox1, and promoting the expression of $\mathrm{Nrf2}$ and SOD2, which demonstrated that isoquercitrin might promote nerve regeneration and functional recovery by inhibiting oxidative stress.

\section{Conclusions}

The current study demonstrated that isoquercitrin might promote nerve regeneration and functional recovery by inhibiting oxidative stress (Figure 9), which extracted the feasibility of using isoquercitrin as a potential neuroprotective drug for peripheral nerve repair applications. However, numerous in-depth studies, such as 


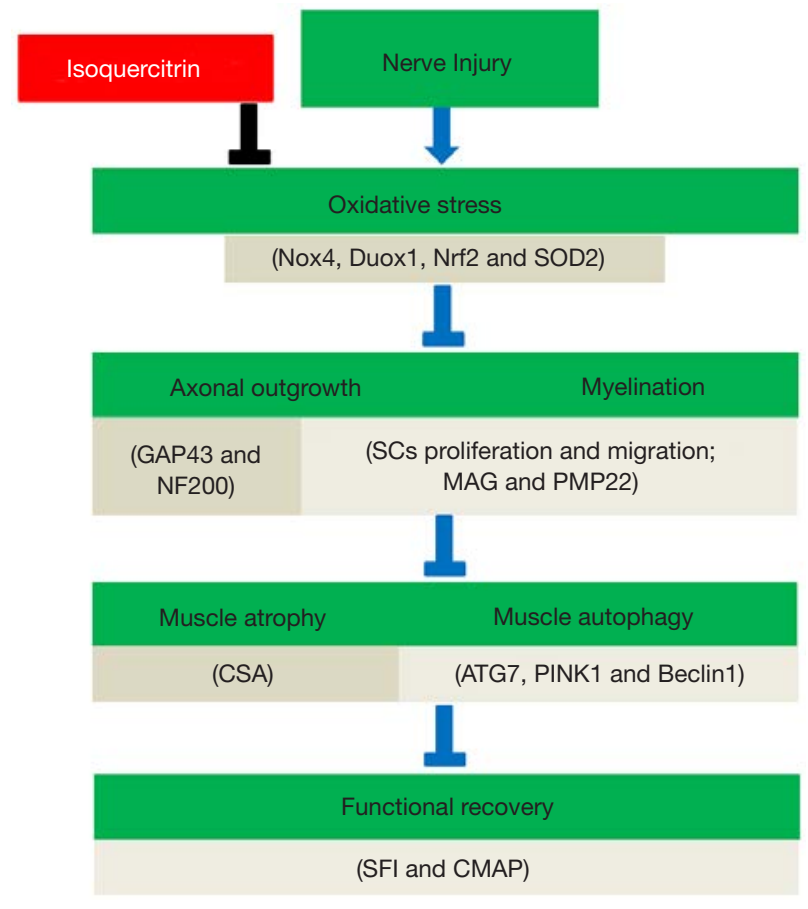

Figure 9 A scheme for isoquercitrin promoting nerve regeneration and functional recovery, and alleviating muscle atrophy by inhibiting oxidative stress.

the precise molecular mechanisms, still need to be pursued before application of isoquercitrin in clinical settings.

\section{Acknowledgments}

Funding: This work was supported by the National Natural Science Foundation of China (Grant No. 81671230, $81871554,31730031,81301628)$, a project funded by Jiangsu Provincial Key Medical Center, the Priority Academic Program Development of Jiangsu Higher Education Institutions (PAPD).

\section{Footnote}

Conflicts of Interest: The authors have no conflicts of interest to declare.

Ethical Statement: The authors are accountable for all aspects of the work in ensuring that questions related to the accuracy or integrity of any part of the work are appropriately investigated and resolved. The whole study protocol is approved by the Ethics Committee of Nantong University (No. 20160308-009) and all the procedures were done according to the guidelines of the Animal Care Committee from the Nantong University, Jiangsu Province, China.

\section{References}

1. Willand MP, Nguyen MA, Borschel GH, et al. Electrical Stimulation to Promote Peripheral Nerve Regeneration. Neurorehabil Neural Repair 2016;30:490-6.

2. Lavasani M, Thompson SD, Pollett JB, et al. Human muscle-derived stem/progenitor cells promote functional murine peripheral nerve regeneration. J Clin Invest 2014;124:1745-56.

3. Petrova V, Eva R. The Virtuous Cycle of Axon Growth: Axonal Transport of Growth-Promoting Machinery as an Intrinsic Determinant of Axon Regeneration. Dev Neurobiol 2018;78:898-925.

4. Attwell CL, van Zwieten M, Verhaagen J, et al. The Dorsal Column Lesion Model of Spinal Cord Injury and Its Use in Deciphering the Neuron-Intrinsic Injury Response. Dev Neurobiol 2018;78:926-51.

5. Yi S, Xu L, Gu X. Scaffolds for peripheral nerve repair and reconstruction. Exp Neurol 2019;319:112761.

6. Jessen KR, Mirsky R. The Success and Failure of the Schwann Cell Response to Nerve Injury. Front Cell Neurosci 2019;13:33.

7. McLachlan EM, Hu P. Inflammation in dorsal root ganglia after peripheral nerve injury: effects of the sympathetic innervation. Auton Neurosci 2014;182:108-17.

8. Shi G, Shi J, Liu K, et al. Increased miR-195 aggravates neuropathic pain by inhibiting autophagy following peripheral nerve injury. Glia 2013;61:504-12.

9. Komirishetty P, Areti A, Yerra VG, et al. PARP inhibition attenuates neuroinflammation and oxidative stress in chronic constriction injury induced peripheral neuropathy. Life Sci 2016;150:50-60.

10. Wang $X$, Zhang G, Qiao Y, et al. Crocetin attenuates spared nerve injury-induced neuropathic pain in mice. J Pharmacol Sci 2017;135:141-7.

11. Xiang Q, Yu C, Zhu YF, et al. Nuclear factor erythroid 2-related factor 2 antibody attenuates thermal hyperalgesia in the dorsal root ganglion: Neurochemical changes and behavioral studies after sciatic nerve-pinch injury. Injury 2016;47:1647-54.

12. Zhang L, Johnson D, Johnson JA. Deletion of Nrf2 impairs functional recovery, reduces clearance of myelin 
debris and decreases axonal remyelination after peripheral nerve injury. Neurobiol Dis 2013;54:329-38.

13. Siqueira Mietto B, Kroner A, Girolami EI, et al. Role of IL-10 in Resolution of Inflammation and Functional Recovery after Peripheral Nerve Injury. J Neurosci 2015;35:16431-42.

14. Lanza C, Raimondo S, Vergani L, et al. Expression of antioxidant molecules after peripheral nerve injury and regeneration. J Neurosci Res 2012;90:842-8.

15. Wang H, Ding XG, Li SW, et al. Role of oxidative stress in surgical cavernous nerve injury in a rat model. J Neurosci Res 2015;93:922-9.

16. Qian Y, Han Q, Zhao X, et al. 3D melatonin nerve scaffold reduces oxidative stress and inflammation and increases autophagy in peripheral nerve regeneration. J Pineal Res 2018;65:e12516.

17. Renno WM, Benov L, Khan KM. Possible role of antioxidative capacity of (-)-epigallocatechin-3-gallate treatment in morphological and neurobehavioral recovery after sciatic nerve crush injury. J Neurosurg Spine 2017;27:593-613.

18. Ydens E, Demon D, Lornet G, et al. Nlrp6 promotes recovery after peripheral nerve injury independently of inflammasomes. J Neuroinflammation 2015;12:143.

19. Zhu M, Li J, Wang K, et al. Isoquercitrin Inhibits Hydrogen Peroxide-Induced Apoptosis of EA.hy926 Cells via the PI3K/Akt/GSK3beta Signaling Pathway. Molecules 2016;21:356.

20. Valentová K, Vrba J, Bancirova M, et al. Isoquercitrin: pharmacology, toxicology, and metabolism. Food Chem Toxicol 2014;68:267-82.

21. Li X, Jiang Q, Wang T, et al. Comparison of the Antioxidant Effects of Quercitrin and Isoquercitrin: Understanding the Role of the 6"-OH Group. Molecules 2016;21. doi: 10.3390/molecules21091246.

22. Hassan W, Rongyin G, Daoud A, et al. Reduced oxidative stress contributes to the lipid lowering effects of isoquercitrin in free fatty acids induced hepatocytes. Oxid Med Cell Longev 2014;2014:313602.

23. Xie W, Wang M, Chen C, et al. Hepatoprotective effect of isoquercitrin against acetaminophen-induced liver injury. Life Sci 2016;152:180-9.

24. Wang CP, Li JL, Zhang LZ, et al. Isoquercetin protects cortical neurons from oxygen-glucose deprivationreperfusion induced injury via suppression of TLR4NF-small ka, CyrillicB signal pathway. Neurochem Int 2013;63:741-9.

25. Wang CP, Shi YW, Tang M, et al. Isoquercetin
Ameliorates Cerebral Impairment in Focal Ischemia Through Anti-Oxidative, Anti-Inflammatory, and AntiApoptotic Effects in Primary Culture of Rat Hippocampal Neurons and Hippocampal CA1 Region of Rats. Mol Neurobiol 2017;54:2126-42.

26. Sun H, Liu J, Ding F, et al. Investigation of differentially expressed proteins in rat gastrocnemius muscle during denervation-reinnervation. J Muscle Res Cell Motil 2006;27:241-50.

27. Huang $\mathrm{SH}, \mathrm{Xu} M, \mathrm{Wu} H M$, et al. Isoquercitrin Attenuated Cardiac Dysfunction Via AMPKalpha-Dependent Pathways in LPS-Treated Mice. Mol Nutr Food Res 2018;62:e1800955.

28. Cibiček N, Roubalova L, Vrba J, et al. Protective effect of isoquercitrin against acute dextran sulfate sodium-induced rat colitis depends on the severity of tissue damage. Pharmacol Rep 2016;68:1197-204.

29. Qiu J, Wang L, Wang Y, et al. MicroRNA351 targeting TRAF6 alleviates dexamethasone-induced myotube atrophy. J Thorac Dis 2018;10:6238-46.

30. Wood MD, Kemp SW, Weber C, et al. Outcome measures of peripheral nerve regeneration. Ann Anat 2011;193:321-33.

31. Pronker MF, Lemstra S, Snijder J, et al. Structural basis of myelin-associated glycoprotein adhesion and signalling. Nat Commun 2016;7:13584.

32. Tae HJ, Rahman MM, Park BY. Temporal and spatial expression analysis of peripheral myelin protein 22 (Pmp22) in developing Xenopus. Gene Expr Patterns 2015;17:26-30.

33. Zhao HT, Damle S, Ikeda-Lee K, et al. PMP22 antisense oligonucleotides reverse Charcot-Marie-Tooth disease type $1 \mathrm{~A}$ features in rodent models. J Clin Invest 2018;128:359-68.

34. Flamm AG, Zerko S, Zawadzka-Kazimierczuk A, et al. $1 \mathrm{H}, 15 \mathrm{~N}, 13 \mathrm{C}$ resonance assignment of human GAP-43. Biomol NMR Assign 2016;10:171-4.

35. Drummond ES, Dawson LF, Finch PM, et al. Increased bilateral expression of alpha1-adrenoceptors on peripheral nerves, blood vessels and keratinocytes does not account for pain or neuroinflammatory changes after distal tibia fracture in rats. Neuroscience 2014;281:99-109.

36. Rosales GJ, Busolini FI, Mohamed FH, et al. Effects of melatonin and gonadal androgens on cell proliferation in the pituitary of viscachas (Lagostomus maximus maximus). Cell Prolif 2016;49:644-53.

37. Feng C, Zhang Y, Yang M, et al. Oxygen-Sensing Nox4 Generates Genotoxic ROS to Induce Premature 
Senescence of Nucleus Pulposus Cells through MAPK and NF-kappaB Pathways. Oxid Med Cell Longev 2017;2017:7426458.

38. Fortunato RS, Gomes LR, Munford V, et al. DUOX1 Silencing in Mammary Cell Alters the Response to Genotoxic Stress. Oxid Med Cell Longev 2018;2018:3570526.

39. Zeng J, Chen Y, Ding R, et al. Isoliquiritigenin alleviates early brain injury after experimental intracerebral hemorrhage via suppressing ROS- and/or NF-kappaBmediated NLRP3 inflammasome activation by promoting Nrf2 antioxidant pathway. J Neuroinflammation 2017;14:119.

40. Katwal G, Baral D, Fan X, et al. SIRT3 a Major Player in Attenuation of Hepatic Ischemia-Reperfusion Injury by Reducing ROS via Its Downstream Mediators: SOD2, CYP-D, and HIF-1alpha. Oxid Med Cell Longev 2018;2018:2976957.

41. Shaw PX, Sang A, Wang Y, et al. Topical administration of a Rock/Net inhibitor promotes retinal ganglion cell survival and axon regeneration after optic nerve injury. Exp Eye Res 2017;158:33-42.

42. Tamaddonfard E, Farshid AA, Samadi F, et al. Effect of vitamin B12 on functional recovery and histopathologic changes of tibial nerve-crushed rats. Drug Res (Stuttg) 2014;64:470-5.

43. Spence JD, Yi Q, Hankey GJ. B vitamins in stroke prevention: time to reconsider. Lancet Neurol 2017;16:750-60.

44. Yuan Y, Shen H, Yao J, et al. The protective effects of Achyranthes bidentata polypeptides in an experimental model of mouse sciatic nerve crush injury. Brain Res Bull 2010;81:25-32.

45. Daeschler SC, Harhaus L, Bergmeister KD, et al. Clinically Available Low Intensity Ultrasound Devices do not Promote Axonal Regeneration After Peripheral Nerve Surgery-A Preclinical Investigation of an FDA-Approved Device. Front Neurol 2018;9:1057.

Cite this article as: Qiu J, Yang X, Wang L, Zhang Q, Ma W, Huang Z, Bao Y, Zhong L, Sun H, Ding F. Isoquercitrin promotes peripheral nerve regeneration through inhibiting oxidative stress following sciatic crush injury in mice. Ann Transl Med 2019;7(22):680. doi: 10.21037/atm.2019.11.18
46. van Neerven SGA, Haastert-Talini K, Boecker A, et al. Two-component collagen nerve guides support axonal regeneration in the rat peripheral nerve injury model. J Tissue Eng Regen Med 2017;11:3349-61.

47. Hung CC, Lin CH, Chang H, et al. Astrocytic GAP43 Induced by the TLR4/NF-kappaB/STAT3 Axis Attenuates Astrogliosis-Mediated Microglial Activation and Neurotoxicity. J Neurosci 2016;36:2027-43.

48. Palazzolo G, Horvath P, Zenobi-Wong M. The flavonoid isoquercitrin promotes neurite elongation by reducing RhoA activity. PLoS One 2012;7:e49979.

49. Hung HA, Sun G, Keles S, et al. Dynamic regulation of Schwann cell enhancers after peripheral nerve injury. J Biol Chem 2015;290:6937-50.

50. Juricek L, Carcaud J, Pelhaitre A, et al. AhR-deficiency as a cause of demyelinating disease and inflammation. Sci Rep 2017;7:9794.

51. Shin YK, Jang SY, Yun SH, et al. Cooperative interaction of hepatocyte growth factor and neuregulin regulates Schwann cell migration and proliferation through Grb2associated binder-2 in peripheral nerve repair. Glia 2017;65:1794-808.

52. Lindborg JA, Mack M, Zigmond RE. Neutrophils Are Critical for Myelin Removal in a Peripheral Nerve Injury Model of Wallerian Degeneration. J Neurosci 2017;37:10258-77.

53. Passipieri JA, Dienes J, Frank J, et al. Adipose Stem Cells Enhance Nerve Regeneration and Muscle Function In A Peroneal Nerve Ablation Model. Tissue Eng Part A 2019. [Epub ahead of print].

54. Milan G, Romanello V, Pescatore F, et al. Regulation of autophagy and the ubiquitin-proteasome system by the FoxO transcriptional network during muscle atrophy. Nat Commun 2015;6:6670.

55. Qiu J, Fang Q, Xu T, et al. Mechanistic Role of Reactive Oxygen Species and Therapeutic Potential of Antioxidants in Denervation- or Fasting-Induced Skeletal Muscle Atrophy. Front Physiol 2018;9:215. 